\title{
RESTRAT, a decision-aiding methodology for restoration of radioactively contaminated sites
}

\author{
Th. Zeevaert, J.P. Hedemann, A. Bousher, V. Brendler and S. Nordlinder
}

SCK-CEN, Boeretang 200, $2400 \mathrm{Mol}$, Belgium

\begin{abstract}
A methodology for evaluating and ranking restoration strategies for radioactively contaminated sites has been elaborated in a project (RESTRAT) under the Fourth Framework of the Nuclear Fission Safety Programme of the EU. The decision-aiding tool developed in this project was based on the principles of justification and optimisation of radiation protection. A multi-attribute utility (MAU) type of analysis was applied in order to be able to deal with social factors, next to radiological health and economic cost attributes. Potentially relevant restoration techniques were identified and their characteristics determined through a literature review. The decision-aiding tool developed has been illustrated by application to representative examples of different categories of contaminated sites. In this paper the results for a contaminated freshwater riverine site are shown.
\end{abstract}

\section{INTRODUCTION}

When nuclear facilities have reached the end of their operational life, the technical part of the installations will be subjected to a controlled decommissioning. However, the sites themselves may require restoration where the contamination has been dispersed or, where radioactive residues are contained by methods that are unreliable for long-term storage.

In the RESTRAT project a transparent, generic decision-aiding methodology has been established for the evaluation and ranking of restoration strategies for radioactively contaminated sites and their close surroundings [1]. A manual has been produced [2]. The project has been partly funded by the EU under the Fourth Framework of the Nuclear Fission Safety Programme.

\section{METHOD}

The method proposed for the evaluation and ranking of restoration options, developed in the RESTRAT project, is based on the radiation protection principles of justification and optimisation, as recommended in ICRP 60 [3].

In this project multiple criteria are being taken into account. They are divided into three major categories: health attributes, economic costs and social factors.

Social factors are difficult to quantify. Therefore, a multi-attribute utility (MAU) analysis is the obvious technique to be applied for optimisation. In order to carry out the evaluations in this way a hierarchy of attributes must be set up. The major attributes are divided into sub-attributes according to the scheme in Figure 1.

In a MAU analysis, each option is assigned a score equal to the sum of the weighted utility values for all attributes [4]. Options with a higher score are preferred to options with a lower score.

The utility values of the attributes for each option are determined through the use of utility functions. In this study, risk-neutral, linear utility functions are applied. These are expressed as:

$U(x)=100\left(1+\frac{x_{\min }-x}{x_{\max }-x_{\min }}\right)$

where $\left(\mathrm{x}_{\min } ; \mathrm{x}_{\max }\right)$ is the value range of the attribute considered. 


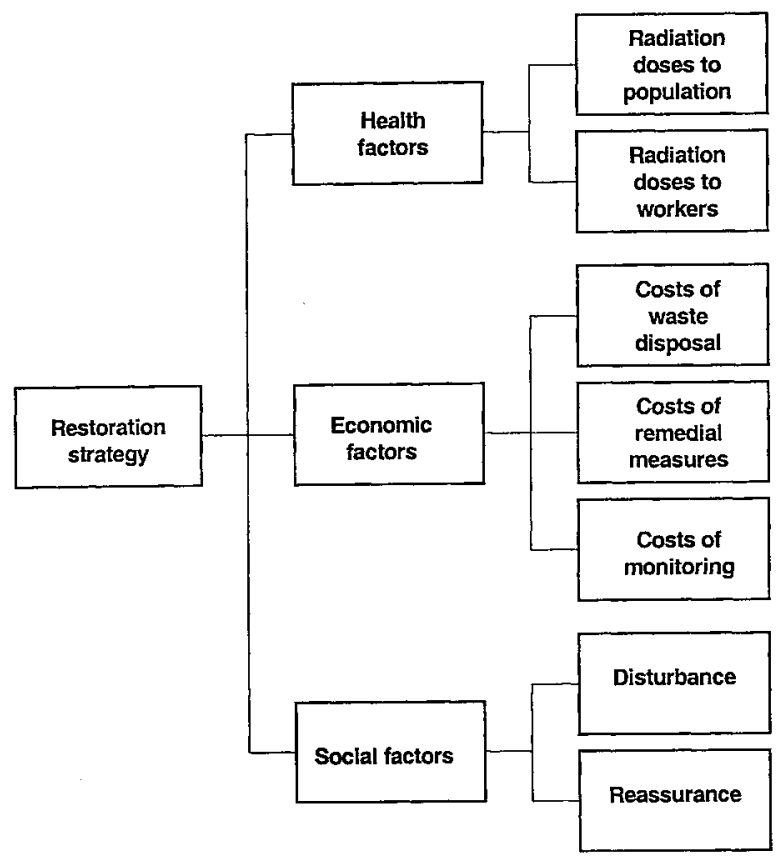

Figure 1: Attribute hierarchy for restoration of a contaminated site

The weighting factors of the attributes, expressing the relative importance of that attribute with respect to the other attributes of the same group, have been determined through the use of a simple conversion method establishing scaling constants between those factors. Expressing these constants for the major attributes as:

$\frac{w_{\text {economic }}}{w_{\text {health }}}=C_{1} \quad \frac{w_{\text {social }}}{w_{\text {health }}}=C_{2}$

the weighting factors for these attributes can be determined by equating their sum with zero.

The value of $C_{1}$ can be determined as the ratio of the ranges of economic costs to the ranges of collective doses, multiplied with the monetary value of the man.Sievert (taken here as 100,000 EUR $\left.\operatorname{manSv}^{-1}\right)$ [5].

The ratio $\mathrm{C}_{2}$ is much more difficult to quantify. Intuitively, it would be expected to be less than one and, for non-accidental situations such as restoration of contaminated sites with small exposures, significantly less than one. In this study a value of 0.25 has been proposed.

The weighting factors for the sub-attributes of a same group have been determined in a similar manner.

\section{ASSESSMENT OF ATTRIBUTES}

\subsection{Radiological Health}

The radiological impact was assessed in terms of effective doses to the public and to the restoration workers. For the public the collective doses have been truncated at 100 and 500 years.

For restoration workers, doses can be calculated straightforward from the contamination levels at the site and the labour volumes required, exposure taking place only through external irradiation and inhalation of resuspended dust.

For the public, a more complicated biosphere model is needed for assessing the doses. A compartmental type of model has been applied, based on the BIOPATH model developed by Studsvik [6]. In this type of model, exchanges or transfers of contamiants between compartments are 
mathematically expressed by a set of linear differential equations. The compartmental scheme applied to the example riverine site is shown in Figure 2.
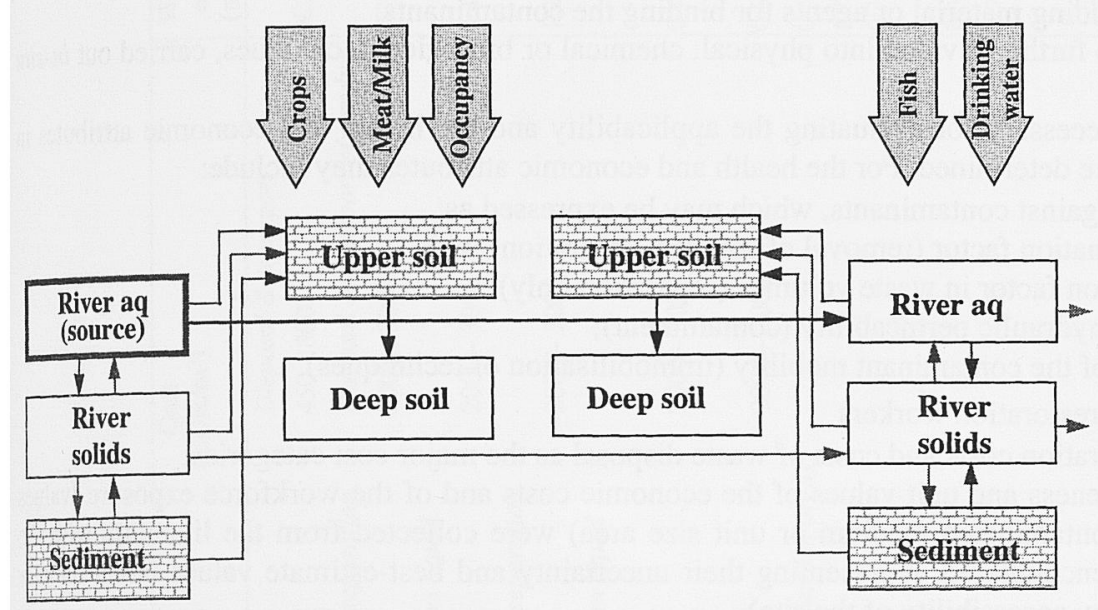

Figure 2: Compartmental scheme for the riverine site

\subsection{Economic costs}

The most important cost categories include:

- pure restoration costs;

- waste disposal costs;

- survey and monitoring costs.

Cost values of the former two categories, used in the example case considered, were derived from the unit cost data, collected from a literature survey (see further, Table 1). Survey and monitoring costs however cannot be derived from literature data. They are very site-specific and have to be derived from sitespecific data.

\subsection{Social factors}

Social factors are non-radiological protection factors. They are not easily quantifiable and vary markedly between countries.

Important social factors that have been considered in this study are:

- reassurance provided by the implementation of remedial measures;

- disturbance caused by the implementation of measures.

In order to quantify these attributes, they have been linked, in this study, to other factors considered to be underlying these attributes. Reassurance has been linked to the residual dose and fraction of activity remaining on the site; its utility values were taken to be inversely proportional to these dose and activity. Disturbance has been linked to the volume of waste removed from the site; its utility values were taken to be inversely proportional to this volume.

\section{CHARACTERISATION OF RESTORATION TECHNIQUES}

Potentially relevant restoration techniques have been identified from a comprehensive literature review. Techniques were selected which were reported as having been applied to remediate radionuclide contamination on medium-sized sites and which were documented with adequate data to allow a good characterisation.

The restoration technologies selected fell into four major categories: 
- Removal of sources: elimination of contaminated media (soil, sediment or water).

- Separation of contaminated fractions.

- Containment: providing barriers around contaminated medium.

- Immobilisation: adding material or agents for binding the contaminants.

These categories were further divided into physical, chemical or biological techniques, carried out in-sith or ex-situ. Table 1

Characteristics, necessary for evaluating the applicability and the health and economic attributes in the MAU analysis were determined. For the health and economic attributes they include:

- the effectiveness against contaminants, which may be expressed as:

$\checkmark$ a decontamination factor (removal of sources, separation) with

- a reduction factor in waste volumes (separation only);

$\checkmark$ a change in hydraulic permeability (containment);

$\checkmark$ a reduction of the contaminant mobility (immobilisation of techniques).

- exposure times of restoration workers

- costs: proper restoration costs and costs of waste disposal as the major cost categories.

Values of the effectiveness and unit values of the economic costs and of the workforce exposure (values per unit volume of contaminated medium or unit size area) were collected from the literature with the ranges of the values encountered, representing their uncertainty and best-estimate values based on sitespecific conditions (e.g. accessibility of the site).

\section{RESULTS AND DISCUSSION}

The ranking methodology for restoration options has been applied to some major categories of contaminated sites. The case study shown here is that of a contaminated riverine site. The nonradiological characteristics of the site were based on those of the Molse Nete river, however the radiological ones have been taken arbitrarily.

The collective effective committed doses to the general public, truncated at 100 and $500 \mathrm{y}$; have been assessed. They are the result of very conservative assumptions concerning actual and future exposures. The exposure pathways were given before.(Figure 2) Utility values and weighting factors of the attributes were derived according to the procedure described above.

The overall scores of the restoration options, are shown in Figure 3. The figure on the left-hand site gives the results for the integration of the collective dose over 100 years; the other figure, the results for an integration time of 500 years. The error bars represent the confidence intervals between the $5^{\text {th }}$ and $95^{\text {th }}$ percentiles of the distribution of the total scores. They were determined by applying the Latin Hypercube sampling technique with the weighting factors and attribute (utility) values sampled from a triangular distribution between $1.5^{-1}$ and 1.5 times the most probable value. Economic costs are clearly dominating.

When considering the best-estimate values of the scores, for the integration of collective dose over 100 years, the no-restoration option seems to be the optimal solution and for the integration over 500 years, capping seems to be the optimal one. However when the uncertainty ranges are taken into account, there is no significant difference between the no-restoration, the containment and (most of) the immobilisation options.

Comparison of the maximum annual dose to the critical group (before restoration) with the IAEA criteria on contaminated land [7], would have as an outcome that the need for restoration varies from "usually needed" (with constraint) to "sometimes needed" (without constraint). 


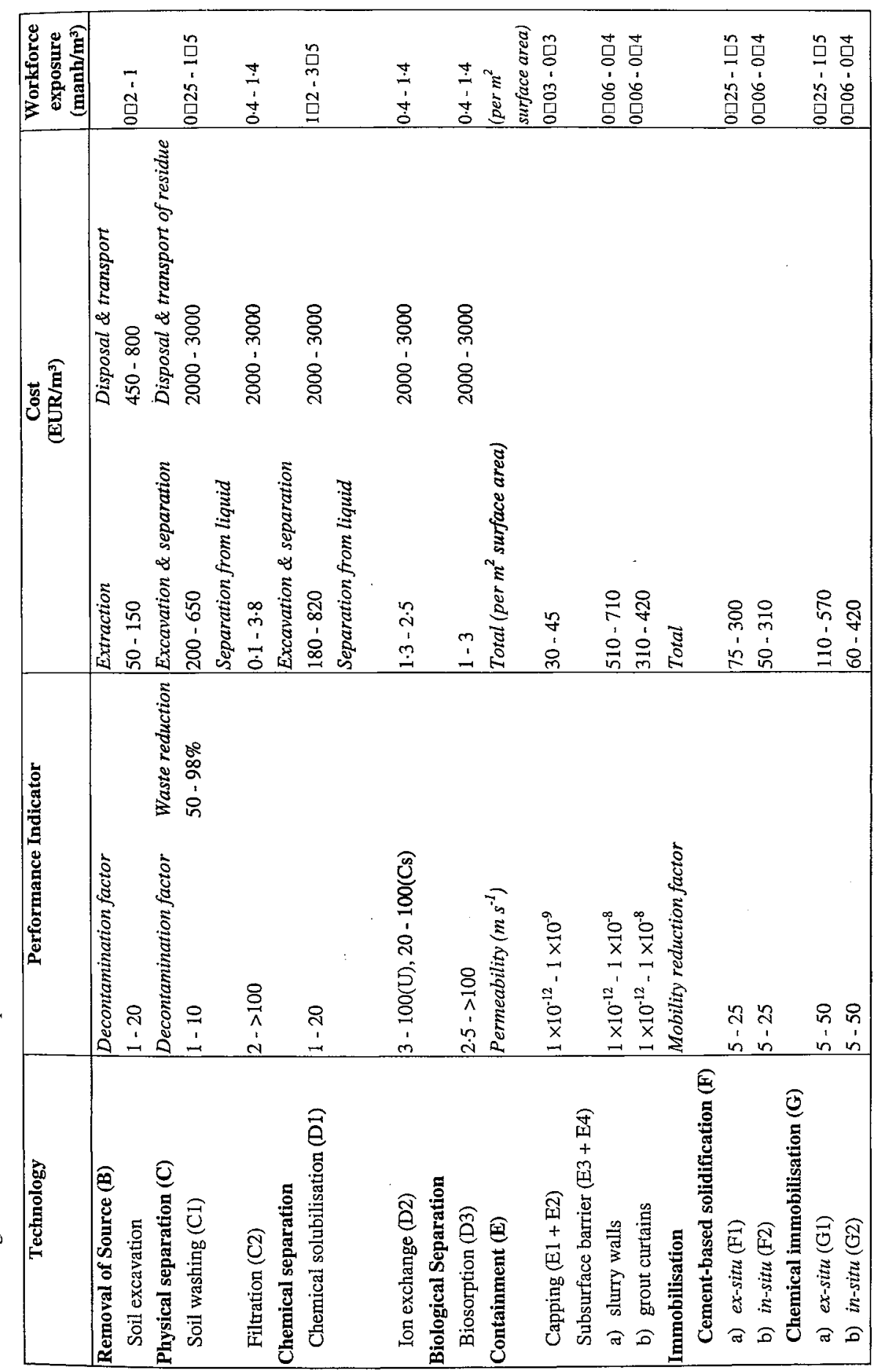



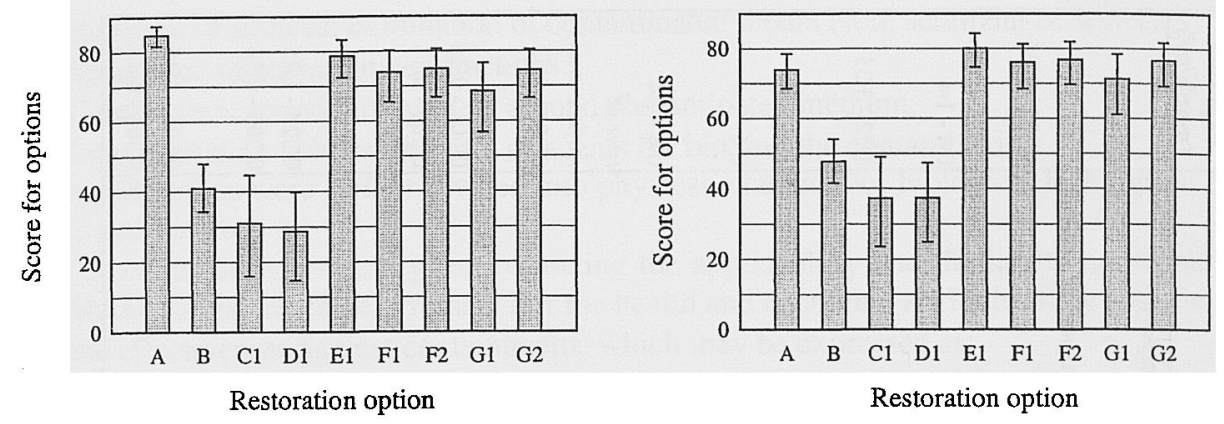

Figure 3: Total scores for restoration options at the riverine site

\section{CONCLUSIONS}

A method for ranking of restoration options for contaminated sites has been elaborated, based on the universal principles of justification and optimisation in radiological protection.

Its usefulness and flexibility has been demonstrated through the application to some examples of major categories of contaminated sites.

Economic costs, in particular waste disposal costs were shown to be in general the dominating attributes for the restoration of sites with low-level contamination. This causes removal of sources (with or without separation) to be never the optimal solution.

\section{REFERENCES}

1. Zeevaert, Th., Bousher, A., Brendler, V., Nordlinder, S., \& Hedemann Jensen, P. RESTRAT Final Report. RESTRAT : Restoration Strategies for radioactively contaminated sites and their close surroundings. SCK·CEN, BLG-822, Mol, Belgium; 1999

2. Zeevaert, Th., Bousher, A., Brendler, V., Nordlinder, S., \& Hedemann Jensen, P. RESTRAT Manual, RESTRAT : Restoration Strategies for radioactively contaminated sites and their close surroundings. SCK·CEN, BLG-819, Mol, Belgium; 1999

3. International Commission on Radiological Protection (ICRP). 1990 Recommendations of the International Commission on Radiological Protection. ICRP Publication 60, Oxford : Pergamon Press, Ann. ICRP 21(1-3); 1991.

4. International Commission on Radiological Protection (ICRP). Optimisation and Decision-Making in Radiological Protection. International Commission on Radiological Protection, ICRP Publication 55, Oxford : Pergamon Press, Ann. ICRP 20 (1); 1989.

5. Nordic Radiation Protection Authorities. The monetary value of collective dose reduction ( $\alpha$-value). Statement from the meeting at Reykjavik, 14 June, 1991. Swedish Radiation Protection Institute, Strälskyddsnytt 4/91, Stockholm, Sweden; 1991.

6. Bergström, U., Edlund, O., Evans, S., \& Röjder, B. BIOPATH - A computer code for calculation of turnover of nuclides in the biosphere and resulting doses to man. Studsvik AB, STUDSVIK/NW. 82/261, Nyköping, Sweden; 1982.

7. International Atomic Energy Agency (IAEA). Application of radiation protection principles to the clean-up of contaminated areas. International Atomic Energy Agency, IAEA - TECDOC - 98\%, Vienna, Austria; 1997. 